\title{
Photometric study of the close binary system DD Monocerotis ${ }^{\star}$
}

\author{
Shengbang Qian, Qingyao Liu, Yulan Yang, Shenghong Gu, and Zhangkui Huang \\ Yunnan Observatory, Academia Sinica, Kunming, China \\ e-mail: bily@public.km.yn.cn
}

Received November 20, 1996; accepted January 30, 1997

\begin{abstract}
New $B V$ light curves of the short-period eclipsing binary system DD Mon have been obtained. Light-curve variability is seen in both $B$ and $V$ bands as compared with the light curves obtained in 1986 by Yamasaki et al. (1990). The light curves are analyzed by using Wilson-Devinney's synthetic light-curve program, and the present photometric solution reveals that DD Mon is a near-contact binary with the secondary component filling the Roche lobe. Combined with Yamasaki et al.'s (1990) spectroscopic results, absolute quantities of DD Mon are derived: mass of the primary $M_{1}=1.05 \pm 0.08 M_{\odot}$, mass of the secondary $M_{2}=0.47 \pm 0.04 M_{\odot}$, radius of the primary $R_{1}=1.36 \pm 0.04 R_{\odot}$, radius of the secondary $R_{2}=$ $1.03 \pm 0.03 R_{\odot}$. These results show that the components of DD Mon have evolved away from the ZAMS and through a mass-transfer process to the present semi-detached state. The variation in shape of the light curve may be caused by the evolution of the system and the activity of dark spots.
\end{abstract}

Key words: stars: DD Mon — binaries: eclipsing — stars: fundamental parameters

\section{Introduction}

The variability of DD Mon (HD 202319) was discovered by Hoffmeister (1934). Later, Ahnert et al. (1947) determined the orbital period to be 0.567989 days from photographic observations. Wachmann (1968) gave a photographic light curve and revised the ephemeris. The first photoelectric photometric and spectroscopic observations were published by Yamasaki et al. (1990) who assigned

Send offprint requests to: Qian Shenbang

* Tables 1 and 2 are only available electronically at the CDS via anonymous ftp to cdsarc.u-strasbg.fr (130.79.128.5) or via http://cdsweb.u-strasbg.fr/Abstract.html the spectral type of the primary component to be F5. According to Yamasaki et al. (1990), DD Mon is an almost semidetached system with the primary component almost filling its critical Roche lobe, while the secondary is not in contact with its critical lobe. The masses of the components are undermassive for their radii and luminosities, which was explained by mass loss from the system during the course of evolution.

These masses of the components and the photometric disturbances and asymmetries on the light curves make DD Mon a rather interesting system to study. Our observed plan was to study the structure and evolutionary state of this system.

\section{Observations}

Photoelectric observations in $B$ and $V$ bands of DD Mon were made on 7 nights in January and February 1996, with the 1.0-m telescope at Yannan Observatory, Academia Sinica. An EMI 6256B photomultiplier was used in the observations. The $B$ and $V$ filters approximate Johnson's standard $U B V$ system. An integration time of 20 seconds was chosen for both stars and sky. HD 292321 and HD 48867 were chosen as comparison star and check star, the same as used by Yamasaki et al. (1990). Nightly extinction coefficients were determined from observations of the comparison star. The magnitude differences between the check star and comparison star were constant within probable errors of $\pm 0.012 \mathrm{mag}(V)$ and $\pm 0.013 \mathrm{mag}(B)$ during the observations. A total of 403 points were obtained in yellow and 357 points in blue. Differential magnitudes $V$ and $B$, in the sense of the variable minus the comparison, are presented in Table 1 for $V$ band and Table 2 for $B$ band.

From these observations, three times of primary minima were derived by using the quadratic fitting method, and are listed in Table 3. Yamasaki et al. (1990) did 
not publish their times of light minima. They used Wachmann's light elements to compute the phases of their observations and applied the correction of $-0.108 \mathrm{P}$ in their calculations. In this study, we have calculated a new ephemeris of DD Mon by combining our three times of light minima with the four times of light minima determined from yamasaki et al.'s (1990) observations, as

$$
\begin{aligned}
\text { Min. I }= & \text { HJD2450100.2891 } \pm 17 \\
& +0.56801688 \pm 34 \times E
\end{aligned}
$$

The $\mathrm{O}-\mathrm{C}$ values listed in Table 3 , were calculated with the new ephemeris. The observed times of light minima are too few to study any period change of the system. More observations for DD Mon will be necessary to determine the period behaviour of this system.

The light variations of DD Mon relative to HD 292321 are shown in Fig. 1 as open circles. To compare with the 1985/1986 observations published by Yamasaki et al. (1990), we also show their observations in Fig. 1 as filled circles. It is very clear that the light curves of DD Mon have changed in the time interval between 1985/1986 and 1996. The maximum brightness of our light curves was fainter by $0.03 \mathrm{mag}(B)$ and $0.08 \mathrm{mag}(V)$ and the minimum brightness was also fainter by $0.08 \mathrm{mag}(B)$ and $0.12 \mathrm{mag}(V)$ as compared with the $1985 / 1986$ observations. The photometric asymmetries (O'Connell effect) on Yamasaki et al.'s (1990) light curves are not seen on our light curves.

\section{Photometric solution}

Photometric solutions of DD Mon were obtained by using the 1992 version of the Wilson-Devinney program (Wilson 1992). The present observations were combined into 60 and 55 normal points in $V$ and $B$, and the number of the individuals in each normal point was taken as the weight of each point. DD Mon is a single-lined spectroscopic binary (SB1) (Yamasaki et al. 1990), and so the spectroscopic orbit can not provide the mass ratio $q$ directly. A search for a reliable mass ratio $q$ via the photometric solution is very necessary. As the $B$ observations at secondary minimum is slightly deficient, the single $V$ light curve was employed in deriving the mass ratio $q$. According to the spectrum F5 of DD Mon (Yamasaki et al. 1990), we adopted the temperature of $6600 \mathrm{~K}$ for star 1 (star eclipsed at Min.I). The gravity-darkening $g_{1}=g_{2}=0.32$, the bolometric albedo $A_{1}=A_{2}=0.5$, and the limb-darkening coefficients $x_{1}=0.78, x_{2}=0.84(B)$ and $x_{1}=0.60, x_{2}=0.68(V)$ were assumed. The solutions for several assumed values of mass ratio $q(q=0.3,0.4,0.5,0.6,0.7,0.8,0.9)$ were obtained. The adjustable parameters were: the inclination $i$, the mean temperature of star $2, T_{2}$, the monochromatic luminosity of star $1, L_{1}$, and the dimensionless potentials of star 1 and star $2, \Omega_{1}$ and $\Omega_{2}$.

For all mass ratios the calculation started at mode 2 (detached mode). In the calculation we found that the solution converged to mode 5 (semi-detached mode) when $q=0.3,0.4,0.5$ and 0.6 , and the solution converged to mode 2 when $q=0.7,0.8$ and 0.9 . The resulting sums of weighted square deviations $\sum$ for each value of $q$ are listed in Table 4 and plotted in Fig. 2. A minimum of $\sum$ is achieved at $q=0.5$. Therefore, we chose the initial value of $q$ to be 0.5 and made it an adjustable parameter. Then we performed a differential correction until it converged and found that the mass ratio $q$ converged to $q=0.45 \pm 0.01$. Finally, we fixed the mass ratio $q=0.45$, and used the two-colour $B$ and $V$ observations to derive the photometric parameters of DD Mon as listed in Table 5. Figure 3 shows the theoretical light curves (solid lines) computed with these parameters. Our solution reveals that the system is a semi-detached binary with the secondary component filling its Roche lobe.

\section{Conclusion and discussion}

Our photometric parameters (Table 5) have been combined with Yamasaki et al.'s (1990) spectroscopic elements to obtain absolute values of DD Mon:

$$
\begin{aligned}
\text { Mass of the primary } & M_{1}=1.05 \pm 0.08 M_{\odot} \\
\text { Mass of the secondary } & M_{2}=0.47 \pm 0.04 M_{\odot} \\
\text { Radius of the primary } & R_{1}=1.36 \pm 0.04 R_{\odot} \\
\text { Radius of the secondary } & R_{2}=1.03 \pm 0.03 R_{\odot} \\
\text { Luminosity of the primary } & L_{1}=3.13 \pm 0.18 L_{\odot} \\
\text { Luminosity of the secondary } & L_{2}=0.65 \pm 0.04 L_{\odot} \\
\text { Separation } & A=3.32 \pm 0.10 R_{\odot} .
\end{aligned}
$$

The present solution indicates that DD Mon is a semidetached system with the secondary component filling its Roche lobe. Yamasaki et al.'s (1990) solution indicated a near-semidetached system with the primary component almost filling its Roche lobe. The photometric parameters are also distinctly different, which may be caused by the following reasons: (1) Yamasaki et al. (1990) and we used different analysis method. They used their own program, while we employed Wilson-Devinney's synthetic code. Generally speaking, the two methods should give the same solutions, but, for binaries with some photometric disturbances, the solution often is not unique; there are two minimal values on the $\sum-q$ diagram (Fig. 2), one at $q=0.5$, the other at $q=0.7$. As we can see in Fig. 2, Yamasaki et al.'s solution (at $q=0.7$ ) appear to be local 
Table 3. Times of light minimum for DD Mon

\begin{tabular}{ccccc}
\hline JD.Hel. & Errors(days) & $E$ & $\mathrm{O}-\mathrm{C}$ & Observers \\
2446411.3042 & 0.0012 & -6494.5 & +0.0007 & Yamasaki et al. \\
2446420.1085 & 0.0009 & -6479 & +0.0008 & Yamasaki et al. \\
2446443.1111 & 0.0009 & -6438.5 & -0.0013 & Yamasaki et al. \\
2446443.9643 & 0.0019 & -6437 & -0.0001 & Yamasaki et al. \\
2450100.2908 & 0.0004 & 0 & +0.0017 & present \\
2450124.1447 & 0.0004 & 42 & -0.0011 & present \\
2450128.1213 & 0.0004 & 49 & -0.0006 & present \\
\hline
\end{tabular}

Table 4. The mass ratio $q$ and corresponding residual $\sum W_{i}(\mathrm{O}-\mathrm{C})_{i}^{2}$

\begin{tabular}{cccccccc}
\hline$q$ & 0.3 & 0.4 & 0.5 & 0.6 & 0.7 & 0.8 & 0.9 \\
\hline$\Sigma\left(\times 10^{-3}\right)$ & 3.91 & 2.81 & 2.75 & 2.94 & 2.85 & 3.08 & 3.17 \\
\hline
\end{tabular}

Table 5. Photometric solutions for DD Mon

\begin{tabular}{cc}
\hline Parameter & Photometric element \\
$q$ & $0.45 \pm 0.01$ \\
$i$ & $76.5 \pm 0.4$ \\
$L_{1} /\left(L_{1}+L_{2}\right)$ & $0.8452 \pm 0.0034$ \\
$L_{1} /\left(L_{1}+L_{2}\right)$ & $0.8815 \pm 0.0034$ \\
$g_{1}=g_{2}$ & 0.32 \\
$A_{1}=A_{2}$ & 0.5 \\
$\Omega_{1}$ & $2.9797 \pm 0.0093$ \\
$\Omega_{2}$ & 2.7718 \\
$x_{1 B}$ & 0.750 \\
$x_{2 B}$ & 0.810 \\
$x_{1 V}$ & 0.600 \\
$x_{2} V$ & 0.680 \\
$T_{1}$ & $6600 \mathrm{~K}$ \\
$T_{2}$ & $5118 \pm 20 \mathrm{~K}$ \\
$r_{1}($ pole $)$ & $0.3905 \pm 0.0014$ \\
$r_{1}($ point $)$ & $0.4515 \pm 0.0029$ \\
$r_{1}($ side $)$ & $0.4095 \pm 0.0017$ \\
$r_{1}($ back $)$ & $0.4274 \pm 0.0020$ \\
$r_{2}($ pole $)$ & 0.2915 \\
$r_{2}($ point $)$ & 0.4187 \\
$r_{2}($ side $)$ & 0.3041 \\
$r_{2}($ back $)$ & 0.3367 \\
\hline
\end{tabular}

solutions. (2) The variation of light curves may also contribute to the differences in solutions.

Yamasaki et al.'s (1990) absolute parameters derived from their photometric mass ratio $q=0.7 \pm 0.15$ are:

Mass of the primary $\quad M_{1}=0.37 \pm 0.20 M_{\odot}$

Mass of the secondary $M_{2}=0.26 \pm 0.09 M_{\odot}$

Radius of the primary $R_{1}=0.99 \pm 0.18 R_{\odot}$
Radius of the secondary $R_{2}=0.68 \pm 0.11 R_{\odot}$

Separation $A=2.47 \pm 0.37 R_{\odot}$.

These absolute parameters suggest that the components of DD Mon are very undermassive for their radii and luminosities, indicating that the components of DD Mon are giants or subgiants. This is unlikely because the evolution times of components with such small masses are longer than the lifetime of the Galaxy. Yamasaki et al. (1990) proposed that this was caused by mass loss from the system during the course of evolution. Their finding that 
DD Mon is a detached system is difficult to understand. The absolute parameters determined by using our photometric mass ratio provide a mass for the primary component that is sensibly consistent with the observed spectral type of F5V determined by Yamasaki et al. (1990), and demonstrates that the system is a post-mass-transfer semi-detached binary. The locations of the two components in the mass-radius plane and in the HR diagram are consistent with similar systems reported in Hilditch et al. (1988).

Yamasaki et al. and we used the same comparison star and check star. Our magnitude difference between the check star and the comparison star was equal to that of Yamasaki et al., which indicates that the comparison star was not variable in the period between 1985/1986 and 1996. The variation of maximum light and minimum light may be caused by evolution of the system. The photometric asymmetries (O'Connell effect) on Yamasaki et al.'s (1990) light curves were not seen when we observed. This was seen also in the case of SS Ari (Qingyao Liu et al. 1993), which demonstrated that DD Mon belongs to this kind of binary with variable O'Connell effect. The photometric disturbance are presumably caused by the presence of dark spots variable extent.

\section{References}

Ahnert P., Hoffmeister C., Rohlfs E., van de Voode A., 1947, Veroff. Sternw. Sonneberg 1, No. 2

Hilditch R.W., King D.J., McFarlane T.M., 1988, MNRAS 231, 341

Qingyao Liu, et al., 1993, A\&AS 101, 253

Wachmann A.A., 1968, Astron. Abh. Hamburger-Sternw. Bergedorf 7, No. 8

Wilson R.E., 1992, Revised version of Wilson-Devinney code, (private communication)

Yamasaki A., et al., 1990, AJ 99, 1218 